Research Paper

\title{
The Influence of C3435T Polymorphism of the ABCBI Gene on Genetic Susceptibility to Depression and Treatment Response in Polish Population - Preliminary Report
}

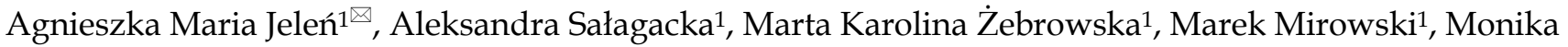
Talarowska², Piotr Gałecki², Ewa Izabela Balcerczak ${ }^{1}$

1. Laboratory of Molecular Diagnostics and Pharmacogenomics, Department of Pharmaceutical Biochemistry and Molecular Diagnostics, Interdepartmental Chair of Laboratory and Molecular Diagnostics, Medical University of Lodz, Poland

2. Department of Adult Psychiatry, Medical University of Lodz, Poland

$\square$ Corresponding author: Agnieszka Maria Jelen, Laboratory of Molecular Diagnostics and Pharmacogenomics, Department of Pharmaceutical Biochemistry and Molecular Diagnostics, Interdepartmental Chair of Laboratory and Molecular Diagnostics, Medical University of Lodz, Muszyńskiego 190-151 Lodz, Poland. phone/fax: +48 4267791 30; e-mail: agnieszka.jelen@umed.lodz.pl

(c) 2015 Ivyspring International Publisher. Reproduction is permitted for personal, noncommercial use, provided that the article is in whole, unmodified, and properly cited. See http://ivyspring.com/terms for terms and conditions.

Received: 2015.07.02; Accepted: 2015.10.11; Published: 2015.11.20

\begin{abstract}
Background: Despite the high prevalence of depression, the mechanism of the origin of this disease as well as the causes of resistance to therapy in some patients are still not fully understood. Increasingly, the possible role of genetic factors is considered. One of them is polymorphisms in the $A B C B I$ (MDRI) gene which encodes P-glycoprotein, responsible for the transport of xenobiotics, including antidepressant drugs, through the blood-brain barrier.

Methods: C3435T was evaluated in 90 patients with recurrent depressive disorders (rDD). Genotyping was performed using a polymerase chain reaction restriction fragment length polymorphism (PCR-RFLP).

Results: The obtained results indicate that the TT genotype occurred more frequently among patients with $r D D$ than in healthy volunteers $(p=0.0441)$. Also, at least one $C$ allele was present significantly less frequent in the study group than in healthy individuals $(p=0.0300)$. The severity of depressive symptoms was higher among patient with the CC genotype in comparison with the other genotypes $(p=0.0106)$ but treatment response to antidepressants was better in this group than among patients with CT or TT genotypes $(p=0.0301)$. Likewise, patients with the T allele have a significantly lower severity of symptoms $(p=0.0026)$ and decreased therapy effectiveness $(p=0.0142)$ than $C$ allele carriers.
\end{abstract}

Conclusions: This study suggests that $C 3435 T$ polymorphisms in the $A B C B I$ gene are strongly associated with a predisposition to depression development, the severity of depressive symptoms and the effectiveness of therapy with using different groups of antidepressant agents.

Key words: $A B C B 1$, single nucleotide polymorphism, P-glycoprotein, pharmacogenetics, depression, antidepressants

\section{Introduction}

Depression is a widespread mental disorder. Many factors predisposing people to the development of depression are known, for example: monoamine deficiency in the brain regions responsible for regulation of mood, hyperactivity of the hypothalam- ic-pituitary-adrenal axis and modifications of the amount or function of brain-derived neurotrophic factor elicited by chronic stress [1], high concentration of proinflammatory cytokines [2] or the possible role of omega-3 polyunsaturated fatty acids [3]. These 
findings not only attempt to clarify the etiology of mood disorders but also find a new target for therapy. Nevertheless, the origination of depression as well as resistance to antidepressant treatment are still not completely understood.

Currently, a great number of drugs from different classes are used in the treatment of depression. However, the available antidepressants give an unsatisfying response rate and cause side effects among treated patients, thus the issue of the causes of treatment failure is still significant [4].

The appropriate level of drugs in the brain is regulated by components of the blood-brain barrier whose primary function is the protection of sensitive cerebral tissues from foreign and neurotoxic substances. One of the best known elements of this barrier is P-glycoprotein (P-gp), a multidrug resistant transporter, which is a 1280 amino acid transporter encoded by $A B C B 1$ (ATP-binding cassette, subfamily $\mathrm{B}$ member 1). P-gp is involved in the active transport of substrates with a diverse chemical structure out of cell. On the one hand, this protein can potentiate the removal of unnecessary endogenous compounds from the brain into the blood circulation. On the other hand, it limits the uptake of exogenous compounds from the blood into the brain. A characteristic feature of this transporter is its broad substrate range [5]. This protein is physiologically expressed in other tissues (e.g. intestine, kidney and placenta epithelial cells or the biliary canalicular membrane of hepatocytes) associated with absorption, distribution, metabolism and excretion of a lot of chemical compounds, including drugs [6]. Furthermore, P-gp plays a part in the regulation of apoptosis, cellular immune response and differentiation of stem-cells.

$A B C B 1$ gene (formerly called MDR1) is located on chromosome 7 (7q21.12) and is highly polymorphic. One of the most extensively studied of the $A B C B 1$ polymorphisms is the silent single nucleotide polymorphism (SNP) C3435T (rs1045642), located in the middle of exon 26, the consequence of which is the alteration of the nucleotide, from cytosine to thymine [5]. It is important to emphasize that the $T$ allele of this polymorphism occurs with a high prevalence in the Caucasian population (about 50\%) and its frequency is influenced by ethnicity [7]. Presumptive medical consequences of its presence may concern a significant proportion of people.

Despite the fact that 3435 polymorphism does not change the amino acid sequence, it have an influence on gene expression and protein formation. Synonymous polymorphism may exert impact on protein function by changing mRNA splicing, folding and stability or modification of translation efficiency. Key role in all mentioned processes play availability of particular codons which is characteristic for each of them. The significance of the polymorphism to producing a predisposition to the development of various diseases and/or resistance to treatment drugs which are substrates of P-gp has been confirmed $[8,9]$. The first to describe the association between C3435T and $A B C B 1$ gene expression in the year 2000 was Hoffmeyer et al [10].

The objective of this study was to determine the association between the occurrence of particular allelic variants of C3435T and the presence of depression, the severity of its symptoms and the efficiency of antidepressant therapy as well as a comparison of received results with data from the literature.

\section{Material and methods}

The study was performed on 90 patients suffering from recurrent depressive disorders (rDD) (32 males and 58 females, mean age at the onset of disease 42.6, range 14-61), which came from the central region of Poland and were diagnosed in the J. Babinski Memorial Psychiatric Hospital in Lodz. The Hamilton Depression Rating Scale (HDRS) is clinician-rated interview used to assess the severity of depressive disorder. The 17-item version of this scale was used to evaluate the severity of depressive symptoms before (HDRS I) and after treatment (HDRS II). The obtained before and during therapy scores, which range from 0 to 54, are a measure of disease and effectiveness of medication. The HDRS test was performed by the same psychiatrist.

Patients were selected for the study according to the inclusion criteria of ICD-10 (F32.0-7.32.2, F33.0-F33.8) [11]. All the subjects were examined during the course of their hospitalization. The presence of axis I and II disorders, other than a depressive episode, and the diagnosis of somatic diseases and injuries of the central nervous system, which could have affected cognitive performance, were regarded as exclusion criteria. Other exclusion criteria were: inflammatory or autoimmune disorders and unwillingness to give informed consent.

In all the included subjects, a case history was obtained prior to the main study procedure, using the standardized Composite International Diagnostic Interview (CIDI) [12]. During hospitalization all the patients received antidepressant pharmacotherapy.

The effectiveness of the therapy was assessed as the change in the HDRS score which was a result of treatment (HDRS change = HDRS I - HDRS II). A detailed characteristic of the investigated group is presented in Table 1. The patients were treated with different types of medicines or a combination of them (18 - selective serotonin reuptake inhibitors (SSRIs), 10 - SSRI with another antidepressant, 14 - venlafaxine of 
the serotonin and norepinephrine reuptake inhibitors (SNRIs), 9 - venlafaxine with another antidepressant, 15 - agomelatine which is antagonist of 5-HT2C and agonist of melatonin MT1 and MT2 receptors, 8 - a combination of other antidepressants). For the other subjects, no information about the course of treatment was available.

Table 1. Clinical characteristic of investigated group

\begin{tabular}{lllllll}
\hline & $\mathrm{n}$ & mean & median & min- max & $\begin{array}{l}\text { quartile } \\
\text { range }\end{array}$ & S.D. \\
\hline $\begin{array}{l}\text { Age at the onset of } \\
\text { depression (years) }\end{array}$ & 90 & 42.6 & 46.0 & $14.0-61.0$ & $36.0-51.0$ & 11.1 \\
HDRS I & 90 & 22.3 & 22.0 & $7.0-37.0$ & $17.0-27.0$ & 6.8 \\
HDRS II & 83 & 7.2 & 7.0 & $0.0-22.0$ & $4.0-10.0$ & 5.0 \\
HDRS change & 83 & 15.3 & 15.0 & $2.0-35.0$ & $10.0-19.0$ & 6.5 \\
\hline
\end{tabular}

The control group consisted of 96 healthy blood donors recruited from the same geographical area as the patients. The data about $A B C B 1$ genotyping which was carried out in the Laboratory of Molecular Diagnostics and Pharmacogenomics was published previously [13].

DNA was isolated from blood samples collected from all subjects. Informed consent was obtained from each participant enrolled in this study. The study was in accordance with the principles of the Declaration of Helsinki and was approved by the Ethical Committee of the Medical University of Lodz (Nr RNN/566/08/KB).

Genomic DNA was isolated from peripheral blood leukocytes according to the standard phenol/chloroform procedure. The SNP C3435T of $A B C B 1$ was genotyped using the polymerase chain reaction restriction fragment length polymorphism (PCR-RFLP) method as reported previously [14].

Statistical analyses were executed using STATISTICA (data analysis software system), version 10 from StatSoft, Inc. (2011) (www.statsoft.com). Genotypes and allele frequencies between the investigated group and healthy volunteers were compared with $\chi^{2}$ test. To research the relationship between the presence of particular genotypes and allele and other clinical features, like age at the onset of depression, gender, value on the HDRS before therapy and change in this scale under treatment, the following were used: ANOVA, Mann-Whitney U test, t-Student test and $\chi^{2}$ test. The level of statistical significance was assumed at $\mathrm{p}<0.05$.

Due to the fact that patients were treated with different drugs from various classes or with combinations of medicines tailored to the individual need of the persons, a statistical analysis of the association between the response to particular drugs among patients and the different genotypes was not possible.

\section{Results}

The frequency of particular genotypes observed and calculated according to the Hardy-Weinberg equilibrium (HWE) was similar. Among patients with rDD, the CC was found in $15(16.7 \%)$, CT in 43 $(47.8 \%)$, and TT in $32(35.6 \%)$ subjects. The frequency of the $\mathrm{T}$ allele in the investigated group was $42.2 \%$. The results obtained previously for the healthy control were as follows: CC in $27(28.1 \%)$, CT in 48 $(50.0 \%)$, TT in 21 (21.9\%) and T allele in $90(46.9 \%)$ controls [13]. When the results obtained in both of these groups were compared, a higher frequency of TT genotype $(p=0.0441)$ and trend towards higher incidence of allele $\mathrm{T}(\mathrm{p}=0.0566)$ among depression cases than controls was observed. Also, the presence of at least one $3435 \mathrm{C}$ allele was significantly higher in the healthy individuals than the study group $(\mathrm{p}=0.0300)$.

In the investigated group, the potential association of SNP C3435T of ABCB1 with age at the onset of disease, gender, severity of depressive symptoms expressed in the HDRS score before therapy, and treatment-related change of HDRS score was evaluated. An association between genotypes and neither age at the time of diagnosis (Table 2) nor gender were stated. CC homozygotes constitute $9.4 \%$ and $20.3 \%$, CT heterozygotes $53.1 \%$ and $44.1 \%$, and TT homozygotes $37.5 \%$ and $35.6 \%$ of men and women, respectively. At least one $\mathrm{T}$ allele was found in $90.6 \%$ of men and $79.7 \%$ of women. Both the HDRS I $(p=0.0106)$ and the HDRS change $(p=0.0301)$ were related to genotype. The mean of HDRS I and the median of the HDRS change were higher among patients with the CC genotype in comparison with other genotypes. Similarly, the mean of HDRS I $(p=0.0026)$ and HDRS change $(p=0.0142)$ was lower in subjects with a T allele than in subjects with a $\mathrm{C}$ allele. Detailed data is included in Table 2.

\section{Discussion}

The main purpose of this study was to evaluate the influence of $A B C B 1 \mathrm{C} 3435 \mathrm{~T}$ on predisposition to the development of depression, the severity of depressive symptoms and the effectiveness of therapy with using different groups of antidepressant agents. Analysis included samples obtained from 90 patients with rDD. The presented study showed that this polymorphism has a proven impact on all of the mentioned elements. 
Table 2. C3435T $A B C B I$ genotype and allele frequencies in relation to age at the onset of depression, severity of symptoms in HDRS score (HDRS I) and effectiveness of therapy as a change in HDRS score after treatment in depressed patients

\begin{tabular}{|c|c|c|c|c|c|c|}
\hline \multirow{2}{*}{$\begin{array}{l}A B C B 13435 \\
\text { Genotype }\end{array}$} & \multicolumn{6}{|c|}{ age at the onset of depression 1} \\
\hline & $\mathrm{n}$ & median & $\min$. & $\max$. & $\begin{array}{l}\text { quartile } \\
\text { range }\end{array}$ & $\mathrm{p}$ value \\
\hline CC & 15 & 44.0 & 25.0 & 61.0 & $40.0-51.0$ & $0.9819^{\wedge \wedge}$ \\
\hline CT & 43 & 46.0 & 20.0 & 55.0 & $35.0-51.0$ & \\
\hline TT & 32 & 46.0 & 14.0 & 58.0 & $35.5-52.0$ & \\
\hline $\mathrm{C}$ allele & $\mathrm{n}$ & median & $\min$. & $\max$ & $\begin{array}{l}\text { quartile } \\
\text { range }\end{array}$ & \\
\hline present & 58 & 46.0 & 20.0 & 61.0 & $37.0-51.0$ & $0.8728^{\wedge}$ \\
\hline absent & 32 & 46.0 & 14.0 & 58.0 & $35.5-52.0$ & \\
\hline $\mathrm{T}$ allele & $\mathrm{n}$ & median & $\min$. & $\max$. & $\begin{array}{l}\text { quartile } \\
\text { range }\end{array}$ & \\
\hline present & 75 & 46.0 & 14.0 & 58.0 & $35.0-52.0$ & $0.8881^{\wedge}$ \\
\hline absent & 15 & 44.0 & 25.0 & 61.0 & $40.0-52.0$ & \\
\hline \multicolumn{7}{|l|}{ HDRS $\mathrm{I}^{2}$} \\
\hline Genotype & $\mathrm{n}$ & mean & $\min$. & $\max$. & SD & \\
\hline CC & 15 & 27.0 & 17.0 & 37.0 & 6.6 & $0.0106^{\wedge \wedge}$ \\
\hline CT & 43 & 21.6 & 10.0 & 32.0 & 6.1 & \\
\hline TT & 32 & 20.5 & 7.0 & 37.0 & 7.7 & \\
\hline C allele & $\mathrm{n}$ & mean & $\min$. & $\max$. & SD & \\
\hline present & 58 & 23.0 & 10.0 & 37.0 & 6.6 & $0.2005^{*}$ \\
\hline absent & 32 & 20.5 & 7.0 & 37.0 & 7.7 & \\
\hline $\mathrm{T}$ allele & $\mathrm{n}$ & mean & $\min$. & $\max$. & SD & \\
\hline present & 75 & 21.4 & 7.0 & 37.0 & 6.4 & $0.0026^{*}$ \\
\hline absent & 15 & 27.0 & 17.0 & 37.0 & 6.6 & \\
\hline \multicolumn{7}{|c|}{ HDRS change $^{3}$} \\
\hline Genotype & $\mathrm{n}$ & median & $\min$. & $\max$. & $\begin{array}{l}\text { quartile } \\
\text { range }\end{array}$ & \\
\hline CC & 13 & 21.0 & 6.0 & 27.0 & $16.0-23.0$ & $0.0301^{\wedge \wedge}$ \\
\hline CT & 41 & 14.0 & 5.0 & 26.0 & $10.0-16.0$ & \\
\hline TT & 29 & 14.0 & 2.0 & 35.0 & $10.0-19.0$ & \\
\hline C allele & $\mathrm{n}$ & mean & $\min$. & $\max$. & SD & \\
\hline present & 54 & 15.8 & 5.0 & 27.0 & 6.0 & $0.3447^{*}$ \\
\hline absent & 29 & 14.4 & 2.0 & 35.0 & 7.3 & \\
\hline $\mathrm{T}$ allele & $\mathrm{n}$ & mean & $\min$. & max. & SD & \\
\hline present & 70 & 14.6 & 2.0 & 35.0 & 6.3 & $0.0142^{*}$ \\
\hline absent & 13 & 19.3 & 6.0 & 27.0 & 6.0 & \\
\hline \multicolumn{7}{|c|}{${ }^{1}$ The age of patients when depression start } \\
\hline $\begin{array}{l}{ }^{2} 17 \text {-item HDI } \\
\text { depressive ep }\end{array}$ & score & or to the & & ation & ring the & nce of \\
\hline
\end{tabular}

Presented in this paper, the frequency of particular genotypes among patients with rDD were similar to those indicated in other studies in New Zealand (CC 21.3\%, CT 51.9\%, TT 26.9\%) [15] or German populations (CC 20\%, CT 60\%, TT 20\%) [16] and different from French (CC $29.1 \%$, CT 47\%, TT 23.9\%) [17] or Japanese populations (CC 33\%, 31\%, 38\%; CT 47\%, $55 \%, 47 \%$; TT $20 \%, 15 \%, 15 \%)[18,19,20]$ with depression. Simultaneously, minor differences in genotypes frequencies were observed between the healthy control and other groups of healthy representatives of Czech (CC 21.1\%, CT 49.9\%, TT 33.9\%) [21], German (CC 27.8\%, 20.8\%; CT 48.3\%, 50.5\%; TT 23.9\%, 28.6\%) $[10,22]$ or British populations (CC $24 \%$, CT $48 \%$, TT
$28 \%$ ) [7]. However, frequencies in Serbian (CC 19\%, CT 54\%, TT 27\%) [23] and Japanese population (CC $35 \%$, CT $50 \%$, TT 15\%) [18] differed widely from the frequencies obtained in the presented study. The frequencies of 3435 genotypes among healthy controls in the others Polish populations were as follows: CC in $41.8 \%, 18.98 \%, 25.4 \%, 23.3 \%$; CT in $41.0 \%, 48.9 \%$, $50.2 \%, 56.3 \%$; TT in $17.2 \%, 32.12 \%, 24.4 \%, 20.4 \%$ [ 14 , $24,25,26]$. Based on these observations it can be concluded that the frequency of C3435T genotypes depends on ethnicity [7]. The different number of individuals in the control groups can be the cause of the discrepancies in results obtained by Polish investigators.

At first, the study showed that the TT genotype occurred significantly more frequently among depressive patients than in controls. A possible association of polymorphism C3435T with a predisposition to development of rDD in the Polish population was found. A similar conclusion was drawn by Fujii et al in the study carried out in the Japanese population [18]. However, Qian et al obtained no association, also in the Japanese population [27], but it should be noted that in this study the recruited subjects suffered from various mood disorders that may result in an inconsistency of conclusions. Because the TT genotype is probably associated with a lower level of expression or change of function of P-gp [10], genotype-driven insufficient activity of the efflux pump leading to accumulation of redundant and toxic substances may contribute to the predisposition to some diseases.

In the present study no correlation of polymorphism C3435T with the age and gender of depressive patients was stated. These findings are consistent with the results obtained by other researchers [17, 20]. However, we observed that patients with the CC genotype obtained a higher value of HDRS I, which means that this genotype may contribute to more pronounced depressive symptoms. In this case, previously mentioned results obtained by Menu et al and Kato et al are dissimilar from ours [17, 20]. Neither French nor Japanese investigators showed the significant association between 3435 genotype and severity of depressive symptoms. The limited number of studies does not allow clear conclusions to be drawn, thus this issue requires further research.

A statistically significant dependence between C3435T genotypes and the effectiveness of antidepressants therapy, measured as a change of the HDRS score which was a result of treatment, was revealed. Patients with the CC genotype obtained a greater change of score, meaning that the effectiveness of therapy among these people was better. The findings published by Menu et al are inconsistent with the results obtained in this study [17]. Published among 
French population study not show an influence the C3435T polymorphism on antidepressant efficacy. These discrepancies may be caused by differences in the drug therapy of the investigated patients. Noordam et al observed that polymorphism C3435T may be associated with switching during therapy, which the authors defined as the necessity of changing an antidepressant drug to any other within the first 45 days of therapy. Patients with TT more frequently required change of administered drug than CC carriers, which may indicate a relationship between this SNP and the tolerance of medicines [28].

Large variations of drugs used in the treatment of the patients in the study did not allow the impact of C3435T on particular antidepressant therapy to be analyzed. However, the data published to date showed that this polymorphism may have an influence on the effectiveness of depression treatment. There is evidence that many antidepressant drugs are substrates for P-gp (e.g. citalopram, paroxetine, and venlafaxine) $[29,30]$. Singh et al demonstrated an association between the dose of drug needed to remission and presence of C3435T among subjects treated with escitalopram. Patients with a $\mathrm{C}$ allele required a greater dose than $\mathrm{T}$ carriers. Also, a better effectiveness of venlafaxine treatment in subjects with the TT genotype compared with CC was observed in this study [31]. Impaired functioning of the transporter in subjects with $\mathrm{T}$ allele presumably allows a higher concentration of the active form of the drug in the cerebrospinal fluid to be maintained, which is necessary to obtain remission. However, Nikish et al did not find a relationship between the presence of 3435 allelic variants and the response to citalopram [16]. Similarly, Kato et al received negative results comparing the effectiveness of paroxetine therapy with the presence of C3435T variants [20].

Changes in effectiveness of treatment may also occur as a result of inhibition or induction of P-gp. Fukui et al observed that the concentration of fluvoxamine in the plasma of psychiatric patients was higher among TT subjects than in the CC genotype group, but only after administration of the highest dose [19]. The reason for these results may be the fact that group of patients with TT only consisted of 4 subjects, thus this study should be replicated in a larger group of patients. On the other hand a great number of P-gp substrates, including fluvoxamine, may exert an inhibitory effect on this transporter [32, 33]. These properties can result in an intensification of activity of other drugs which are P-gp substrates when they are used in combination with a high dose of potential P-gp inhibitor.

Since the influence of C3435T on P-gp expression or its function among depressive patients is still con- troversial, the presence of linkage disequilibrium between this polymorphism and the other $A B C B 1$ SNPs should be taken into consideration. Examples may be found in some studies: Qian et al who found the association of 129T-2677A-3435C haplotype with the occurrence of mood disorders in Japanese patients [27], Kato et al who observed a poor response to paroxetine among subjects with 1236T-2677G-3435C in Japanese patients with major depressive disorder [20].

The first limitation of this study is low number of patients. The second is the usage of different treatment regimens of particular individuals. This did not allow to determine whether 3435 genotype influences on effect of treatment based on P-gp substrates only.

In conclusion, this study of the association of SNP C3435T in the ABCB1 gene with a predisposition to the development of depression in the Polish population was identified. Moreover, the severity of symptoms and effectiveness of antidepressants treatment was found to be connected with this polymorphism. To better understand the causes of such connections, it is desirable to assess other polymorphisms of $A B C B 1$ and to examine the interrelation between them.

\section{Abbreviations}

ABCB1: ATP-binding cassette, subfamily B member 1; CIDI: Composite International Diagnostic Interview; HWE: Hardy-Weinberg equilibrium; HDRS: Hamilton Depression Rating Scale; PCR-RFLP: polymerase chain reaction restriction fragment length polymorphism; P-gp: P-glycoprotein; rDD: recurrent depressive disorders; SNP: single nucleotide polymorphism; SNRI: serotonin and norepinephrine reuptake inhibitor; SSRI: selective serotonin reuptake inhibitor

\section{Acknowledgments}

This study is supported by the Statutory funds of the Department of Pharmaceutical Biochemistry and Molecular Diagnostics, Medical University of Lodz no. 503/3-015-02/503-31-001; Funds of the Faculty of Pharmacy, Medical University of Lodz no. 502-03/3-015-02/502-34-037 and also by the scientific research grants from the National Science Centre no. 2011/01/D/HS6/05484 and no. 2012/05/B/NZ5/ 01452.

\section{Competing Interests}

The authors have declared that no competing interest exists.

\section{References}

[1] Kunugi $\mathrm{H}$, Hori $\mathrm{H}$, Adachi $\mathrm{N}$, et al. Interface between hypothalamic-pituitary-adrenal axis and brain-derived neurotrophic factor in depression. Psychiatry Clin Neurosci. 2010; 64: 447-59. 
[2] Dowlati Y, Herrmann N, Swardfager W, et al. A Meta-Analysis of Cytokines in Major Depression. Biol Psychiatry. 2010; 67: 446-57.

[3] Parker G, Gibson NA, Brotchie H, et al. Omega-3 Fatty Acids and Mood Disorders. Am J Psychiatry. 2006; 163: 969-78.

[4] Fabbri C, Di Girolamo G, Serretti A. Pharmacogenetics of Antidepressant Drugs: An Update After Almost 20 Years of Research. Am J Med Genet B Neuropsychiatr Genet. 2013; 162B: 487-520.

[5] Fung KL, Gottesman MM. A synonymous polymorphism in a common MDR1 (ABCB1) haplotype shapes protein function. Biochim Biophys Acta. 2009; 1794: 860-71.

[6] Lin HJ. Drug-drug interaction mediated by inhibition and induction of P-glycoprotein. Adv Drug Deliv Rev. 2003; 55: 53-81.

[7] Ameyaw MM, Regateiro F, Li T, et al. MDR1 pharmacogenetics: frequency of the C3435T mutation in exon 26 is significantly influenced by ethnicity. Pharmacogenetics. 2001; 11: 217-21.

[8] Siegsmund M, Brinkmann U, Scháffeler E, et al. Association of the P-glycoprotein transporter MDR1 (C3435T) polymorphism with the susceptibility to renal epithelial tumors. J Am Soc Nephrol. 2002; 13: 1847-54.

[9] Kimchi-Sarfaty C, Oh JM, Kim IW, et al. A "silent" polymorphism in the MDR1 gene changes substrate specificity. Science. 2007; 315: 525-8.

[10] Hoffmeyer S, Burk O, von Richter O, et al. Functional polymorphisms of the human multidrug-resistance gene: multiple sequence variations and correlation of one allele with P-glycoprotein expression and activity in vivo. Proc Natl Acad Sci U S A. 2000; 97: 3473-8.

[11] [Internet] WHO, Geneva, Switzerland. The ICD-10 Classification of Mental \& Behavioural Disorders. Diagnostic criteria for research 1993. http://www.who.int/classifications/icd/en/GRNBOOK.pdf.

[12] Patten SB. Performance of the Composite International Diagnostic Interview Short Form for major depression in community and clinical samples. Chronic Dis Can. 1997; 18: 109-12.

[13] Jamroziak K, Balcerczak E, Calka K, et al. Polymorphisms and haplotypes in the multidrug resistance 1 gene (MDR1/ABCB1) and risk of multiple myeloma. Leuk Res. 2009; 33: 332-5.

[14] Jamroziak K, Balcerczak E, Młynarski W, et al. Distribution of allelic variants of functional C3435T polymorphism of drug transporter MDR1 gene in a sample of Polish population. Pol J Pharmacol. 2002; 54: 495-500.

[15] Roberts RL, Joyce PR, Mulder RT, et al. A common P-glycoprotein polymorphism is associated with nortriptyline-induced postural hypotension in patients treated for major depression. Pharmacogenomics J. 2002; 2: 191-6.

[16] Nikisch G, Eap CB, Baumann P. Citalopram enantiomers in plasma and cerebrospinal fluid of $\mathrm{ABCB} 1$ genotyped depressive patients and clinical response: a pilot study. Pharmacol Res. 2008; 58: 344-7.

[17] Menu P, Gressier F, Verstuyft C, et al. Antidepressants and ABCB1 gene C3435T functional polymorphism: a naturalistic study. Neuropsychobiology. 2010; 62: 193-7.

[18] Fujii T, Ota M, Hori $\mathrm{H}$, et al. Association between the functional polymorphism (C3435T) of the gene encoding P-glycoprotein (ABCB1) and major depressive disorder in the Japanese population. J Psychiatr Res. 2012; 46: 555-9.

[19] Fukui N, Suzuki Y, Sawamura K, et al. Dose-dependent effects of the 3435 C $>$ T genotype of ABCB1 gene on the steady-state plasma concentration of fluvoxamine in psychiatric patients. Ther Drug Monit. 2007; 29: 185-9.

[20] Kato M, Fukuda T, Serretti A, et al. ABCB1 (MDR1) gene polymorphisms are associated with the clinical response to paroxetine in patients with major depressive disorder. Prog Neuropsychopharmacol Biol Psychiatry. 2008; 32: 398-404.

[21] Pechandová K, Buzková H, Slanar O, et al. Polymorphisms of the MDR1 gene in the Czech population. Folia Biol (Praha). 2006; 52: 184-9.

[22] Cascorbi I, Gerloff T, Johne A, et al. Frequency of single nucleotide polymorphisms in the P-glycoprotein drug transporter MDR1 gene in white subjects. Clin Pharmacol Ther. 2001; 69: 169-74.

[23] Milojkovic M, Stojnev S, Jovanovic I, et al. Frequency of the C1236T, G2677T/A and C3435T MDR1 gene polymorphisms in the Serbian population. Pharmacol Rep. 2011; 63: 808-14.

[24] Dudarewicz M, Barańska M, Rychlik-Sych M, et al. C3435T polymorphism of the ABCB1/MDR1 gene encoding P-glycoprotein in patients with inflammatory bowel disease in a Polish population. Pharmacol Rep. 2012; 64: 343-50.

[25] Rubiś B, Hołysz H, Barczak W, et al. Study of ABCB1 polymorphism frequency in breast cancer patients from Poland. Pharmacol Rep. 2012; 64: 1560-6.

[26] Droździk M, Białecka M, Myśliwiec $K$, et al. Polymorphism in the P-glycoprotein drug transporter MDR1 gene: a possible link between environmental and genetic factors in Parkinson's disease. Pharmacogenetics. 2003; 13: 259-63.

[27] Qian W, Homma M, Itagaki F, et al. MDR1 gene polymorphism in Japanese patients with schizophrenia and mood disorders including depression. Biol Pharm Bull. 2006; 29: 2446-50.

[28] Noordam R, Aarts N, Hofman A, et al. Association between genetic variation in the ABCB1 gene and switching, discontinuation, and dosage of antidepressant therapy: results from the Rotterdam Study. J Clin Psychopharmacol. 2013; 33: 546-50.

[29] Uhr M, Grauer MT, Holsboer F. Differential enhancement of antidepressant penetration into the brain in mice with abcb1ab (mdr1ab) P-glycoprotein gene disruption. Biol Psychiatry. 2003; 54: 840-6.

[30] Uhr M, Tontsch A, Namendorf C, et al. Polymorphisms in the drug transporter gene ABCB1 predict antidepressant treatment response in depression. Neuron. 2008; 57: 203-9.
[31] Singh $\mathrm{AB}$, Bousman $\mathrm{CA}, \mathrm{Ng} \mathrm{CH}$, et al. ABCB1 polymorphism predicts escitalopram dose needed for remission in major depression. Transl Psychiatry. 2012; 2: e198; doi:10.1038/tp.2012.115.

[32] El Ela AA, Härtter S, Schmitt U, et al. Identification of P-glycoprotein substrates and inhibitors among psychoactive compounds--implications for pharmacokinetics of selected substrates. J Pharm Pharmacol. 2004; 56: 967-75.

[33] Weiss J, Dormann SM, Martin-Facklam M, et al. Inhibition of P-glycoprotein by newer antidepressants. J Pharmacol Exp Ther. 2003; 305: 197-204. 